Présentation de l'ouvrage le replace ainsi dans son contexte : la réponse aux Lettres sur la doctrine de Spinoza, de Jacobi manifeste l'évolution de la pensée de Herder et l'importance que cette pensée a prise pour ses contemporains, qu'ils la critiquent ou s'en inspirent.

Béatrice LENOIR

\title{
HISTOIRE ET RELIGION
}

Robin Cormack, Icônes et société à Byzance. Trad. de l'anglais par Marie-Odile Bernez. Brionne, Gérard Monfort, 1993. 17 × 24, 304 p., ill., bibliogr., index (Imago mundi).

Le présent livre est la traduction d'un ouvrage paru en 1985. Il ne prend pas la forme d'une écriture synthétique, mais d'une suite d'études centrées sur un personnage significatif d'un lieu et d'une époque. De cette façon, s'adressant à un vaste public, il tente de faire revivre concrètement la société byzantine à travers les documents qui sont le mieux conservés, ceux issus de l'art religieux, puisque l'essentiel des archives de cet empire bien administré, mais entièrement disparu, ne nous est pas parvenu. Il part du postulat, à l'évidence exact, que, dans cette société dont une minorité de membres savait lire, le discours oral, par essence perdu, et les arts visuels, relativement bien conservés, jouaient un rôle primordial. Les images Robin Cormack définit au passage l'icône et élargit son propos à toutes les représentations - sont donc essentielles dans la sociêté byzantine, mais leur rôle social évolue dans le temps, ce qui justifie la démarche chronologique. Dans chaque chapitre, Cormack se montre soucieux de nous présenter les grandes évolutions politiques et institutionnelles qui marquent la société étudiée, pour que le lecteur puisse le suivre aisément.

Le premier chapitre, "Le saint visible: saint Théodore de Sykéôn ", nous entraîne dans la Galatie de la fin du vi ${ }^{e}$ et du début du viI ${ }^{e}$ siècle sur les traces d'un saint villageois et de la société qui l'entourait. Cormack commence par présenter le récit hagiographique qui nous fait connaître Théodore. Sa connaissance du dossier hagiographique est ici bien sommaire, car il fait de Théodore « l'un des plus grands (saints) de l'Église byzantine " (p. 22), alors qu'il ne figure pas dans le « top 365 » de la sainteté byzantine, le synaxaire du patriarcat, document qui présentait le ou les saints officiels du jour mentionnés à l'office. Il fait également de la vie écrite au début du vil' siècle par Georges, un disciple du saint, le seul document hagiographique, alors que nous disposons d'un dossier complet comprenant notamment le récit, écrit deux siècles plus tard, du transfert de la relique à Constantinople sur 
l'ordre de l'empereur Héraclius $(610-641)^{1}$. Ces défauts hagiographiques n'empêchent nullement l'auteur de faire une excellente présentation de Théodore dans la société byzantine de cette époque. À travers les mentions, à vrai dire assez peu nombreuses, d'images dans la vie, il montre que celles-ci faisaient normalement partie du décor, qu'elles fournissaient la base matérielle des visions du Christ et de la Vierge dont le saint bénéficie au-delà de l'amélioration du cadre de vie de l'ascète. L'image a ce caractère d'enseignement qui faisait sa fonction d'origine, puisqu'elle rend perceptible le sacré, mais les images sont en partie à la source des pouvoirs surnaturels du saint. L'auteur fait bien sentir la confiance croissante que les fidèles font aux images à cette époque.

Le deuxième chapitre, «Le saint imaginaire: saint Démétrius de Thessalonique ", concerne la même époque, mais se situe dans la plus grande ville de l'Empire en Europe (Rome excepté), alors située sur la ligne de démarcation entre l'Empire et les envahisseurs slaves et avars. Peu de villes ont connu une identification aussi complète entre le martyr qui en est le patron et le destin de la cité; la fête du saint le 26 octobre donne lieu à l'une des foires les plus importantes de l'Empire jusqu'à la chute de la ville aux mains des Turcs au début du $x^{e}$ siècle. Cormack résume la source essentielle que constituent les deux livres de recueils de miracles du saint, où les représentations jouent un rôle éminent; l'église du saint, construite au $v^{e}$ siècle, était en effet couverte de mosaïques qui témoignaient de l'aspect physique du saint et d'une existence historique par ailleurs nullement prouvée. De plus, l'évêque dont l'église constituait la cathédrale était fréquemment représenté au côté du saint et l'image renforçait ainsi son autorité. La présence secourable du saint, ultime et efficace défenseur de la cité, galvanisait les énergies des défenseurs; les icônes portaient aux fidèles témoignage des pouvoirs de Démétrius.

L'auteur consacre le chapitre suivant à un événement central pour son sujet : l'iconoclasme. Là encore, la composition et la démarche sont d'une grande clarté. Il montre d'abord le développement du culte des images au vil ${ }^{e}$ siècle, avec ces étapes décisives que sont le canon du concile de 692 prescrivant de représenter le Christ sous ses traits humains à la place de l'agneau et l'apparition du Christ sur les monnaies durant le règne de Justinien II. Il expose ensuite les difficultés d'interprétation d'une crise où les facteurs politiques et religieux interfèrent, la conception qu'a l'empereur de son rôle religieux y tenant une grande place; l'enjeu, dans les deux camps, c'est la représentabilité du divin, ou plus exactement déterminer si l'on pouvait représenter le divin sous des traits réalistes ou seulement sous des traits symboliques, comme l'hostie ou la croix. Une fois cela posé, Cormack fait de l'iconoclasme une réaction à l'apparition du monothéisme concurrent de l'Islam, à l'éloignement de l'Occident et à la crise des cités, donc au changement de l'environnement général de l'Empire; les deux premières propositions sont largement discutables. Il résume ensuite l'histoire de l'iconoclasme puis se penche longuement sur deux documents : la vie d'Étienne le Jeune, bien étudiée depuis la publication de

1. On trouvera une étude du dossier hagiographique de Théodore de Sykéôn dans Michel KapLAN, "Les sanctuaires de Théodore de Sykéôn", in Les Saints et leur sanctuaire a Byzance. Textes, images et monuments, éd. Catherine Jolivet-Lévy, Michel KaPlan, JeanPierre SodinI, Paris, Publications de la Sorbonne (Byzantina Sorbonensia, 11), 1993, p. 65-79. 
l'ouvrage de Marie-France Auzépy ${ }^{2}$, et la «Lettre des patriarches d'Alexandrie, d'Antioche et de Jérusalem à l'empereur Théophile en l'an 836 ». Ce dernier document assez douteux historiquement, sûrement postérieur à la fin du conflit en 843 et peu étudié de nos jours, se présente comme les actes d'un concile tenu à Jérusalem; il comporte quinze chapitres qui présentent une défense de icônes; l'un d'entre eux, le plus long, consiste en la liste de célèbres icônes miraculeuses, dont les miracles mêmes, conformément à la démarche du concile de Nicée en 787 , sont un argument en faveur du culte des images. L'auteur analyse ensuite les illustrations de plusieurs manuscrits du $\mathrm{IX}^{\mathrm{e}}$ siècle à l'aide des illustrations marginales. De ces études soignées, il conclut que les iconoclastes comme les iconodoules sont portés par une tradition qui pèse d'un poids incomparable dans leur culture. Même si les conclusions sont maintenant quelque peu dépassées par les travaux les plus récents, le point de vue apporté et l'étude des sources sont d'un grand intérêt.

Le chapitre IV reste dans cette ligne : « Après l'iconoclasme : l'illusion de la tradition ». Les premières images sacrées réapparaissent d'abord sur les monnaies; sur les murs des églises, c'est plus graduel et ce n'est pas un simple retour à l'illustration effacée par les iconoclastes. Pour montrer cette restauration des images, Cormack se concentre intelligemment sur le personnage de Photius; il en décrit brièvement la carrière puis cite et commente le texte de l'homélie prononcée de l'ambon de Sainte-Sophie le samedi de Pâques 29 mars 867 pour l'inauguration de la mosaïque de la Théotokos (Mère de Dieu) de l'abside, encore en place. Le grand patriarche exalte le rôle traditionnel de décoration et de didactique des images; la résurrection de l'image naguère disparue est une annonce de la Résurrection. L'auteur examine ensuite les raisons, tues par Photius, de placer la Vierge et l'Enfant à cette place et en vient ainsi à s'étendre sur l'importance à Byzance du culte de la Vierge, qui s'est développé de façon spectaculaire dans la période précédant l'iconoclasme. La façon de la représenter, notamment par l'épisode de la Dormition, qui fait souvent pendant à la Vierge de l'abside dans le programme iconographique, permet de mieux cerner la place qu'elle occupe dans la société et d'expliquer le développement d'un cycle de la Vierge, à côté de celui de la vie du Christ, dans la décoration des églises byzantines, fondé sur des textes scripturaires apocryphes. Ainsi met-on l'accent sur la maternité de la Théotokos et son accessibilité à la prière : la conception théologique le cède devant la vie quotidienne. Peutêtre pourrait-on ajouter à celles de l'auteur une explication plus politique : la Vierge est le défenseur de Constantinople et, partant, de l'Empire; les habitants de la capitale lui vouent depuis longtemps une dévotion passionnée. Quand l'espace pictural est à prendre, au lendemain de l'iconoclasme, elle l'occupe; de là, comme toujours, la mode se répand de Constantinople vers les provinces.

Le chapitre suivant, «La quête du Paradis : l'art au service des empereurs », est plus directement politique; il utilise au premier chef, d'une part les mosaíques encore conservées dans les tribunes de Sainte-Sophie, représentant le Christ entre Zoé et Constantin Monomaque et la Vierge entre Jean II Comnène et Irène, de conserve avec d'autres représentations de ce type, notamment sur les ivoires et dans

2. Marie-France AuzÉPY, La Vie d'Étienne le Jeune, Ashgate, Variorum (Birmingham Byzantine and Ottoman Monographs, 3), 1997. 
les enluminures des manuscrits. Puis il établit le lien avec ce que l'on connaît de la formidable fondation de ces deux derniers souverains, le monastère et hôpital du Pantokratôr, dont l'église triple, encore debout, atteste la splendeur; par les icônes, les empereurs affirment leur emprise sur la société. Enfin, le dernier exemple choisi se situe au tournant du XII et du XIII ${ }^{\mathrm{e}}$ siècle, en Chypre, avec Néophytos le Reclus ( Le Paradis obtenu : l'art privé ») : dans son monastère troglodytique, Néophytos, qui écrit sa propre hagiographie, se fait mettre en scène au beau milieu de l'iconographie.

Pour conclure, Cormack met en avant la place primordiale de l'image dans la société; l'art fournit un cadre à l'intérieur duquel une société ou un individu peut exprimer son idéal. L'icône byzantine n'a pas pour but de représenter par l'artifice une «réalité »: elle exprime la conception du monde qu'ont les Byzantins, bien souvent inexprimée.

Le propos de l'auteur, fermement tenu d'un bout à l'autre de l'ouvrage, est d'un grand intérêt. Celui-ci se trouve soutenu par tout un appareil : liste des empereurs qui permet au lecteur de se situer dans le temps, une carte sommaire pour se situer dans l'espace, un glossaire des termes techniques, une bibliographie commentée pour les ouvrages qui méritent discussion et un index. Ce qu'on attend le plus, ce sont les cent illustrations. Celles-ci sont décevantes : toutes en noir et blanc, pour la plupart situées dans les marges en un format qui les rend illisibles, elles sont accompagnées d'un commentaire historiquement prégnant (encore que représenter sur l'illustration 1 Konya, une ville sur un haut plateau, pour figurer le paysage de Sykéôn, petit village situé au fond d'une vallée encaissée, ne s'impose pas), mais dépourvues des références scientifiques indispensables.

Michel KaPLAN

Louis GIRARD, L'Argument ontologique chez saint Anselme et chez Hegel. Amsterdam/Atlanta, GA, Rodopi, 1995. 15,5 × 23, 664 p., bibliogr. (Elementa, 60).

Important travail, non seulement par son volume, mais aussi par la qualité et l'étendue de l'information, la clarté et la rigueur de l'exposé, que cette étude sur l'argument ontologique. S'il est vrai qu'elle porte surtout sur la conception de cet argument chez Anselme de Canterbury et chez Hegel, c'est cependant l'histoire entière de cette façon d'accéder à Dieu que retrace Louis Girard, de son initiateur Anselme à l'un de ses récents interprètes Yves Cattin, en passant par Bonaventure, Thomas d'Aquin, Duns Scot, Descartes, Malebranche, Spinoza, Leibniz, Hume, Mosheim, Crusius, Kant, Hegel, Schelling, Karl Barth, Norman Malcolm, Charles Hartshorne, Alvin Plantinga, Hick, Mac Gill, Jules Vuillemin, Alexandre Koyré, Paul Vignaux, Michel Corbin. Autant dire qu'il s'agit d'une véritable somme, en ajoutant qu'elle ne se présente pas comme une pure revue des différentes interprétations et critiques de l'argument anselmien qui ont été proposées au cours des siècles. Girard ne porte pas seulement sur les unes et les autres un regard d'historien, il les juge en philosophe, c'est-à-dire en métaphysicien, et met en lumière aussi 
bien leurs faiblesses que leurs côtés positifs. Et, comme il agit de même avec ses deux principaux auteurs, tout son livre est une occasion à la fois de s'instruire sur l'histoire de cet argument et d'exercer sa réflexion sur la dialectique qui le soustend, même si l'on n'est pas toujours d'accord avec son analyse.

Ce qui corrobore qu'il s'agit d'un travail de métaphysicien, en même temps et peut-être davantage, que d'historien, c'est l'ordre d'exposition suivi. Girard part, en effet, de l'argument a priori cartésien, dont il montre, dans l'Introduction, que contrairement à celui d'Anselme basé sur l'unité de Dieu et de l'homme, comme le sera celui de Hegel, il tend à « isoler Dieu dans une transcendance inaccessible » (p. 54). Après quoi, dans la $\mathrm{I}^{\text {re }}$ partie, il présente brièvement l'argument anselmien, puis examine les interprétations et les critiques des auteurs cités à l'instant, avant d'aborder le vif de son sujet dans les $\mathrm{II}^{\mathrm{e}}$ et $\mathrm{II}^{\mathrm{e}}$ parties respectivement consacrées à Anselme et à Hegel.

«Anselme ou la raison qui prie », tel est le titre de la II ${ }^{\mathrm{e}}$ partie (p. 139). Girard y souligne que, pour saisir la pensée de celui-ci sur l'argument ontologique dans sa vérité et sa densité, il faut se garder d'en chercher l'expression uniquement dans les chapitres II, III et IV du Proslogion, et s'attacher à en suivre le cheminement antérieur dans le Monologion et ultérieur dans le Liber apologeticus et le De veritate. Car, explique-t-il, si la démarche du Proslogion, qui consiste dans la recherche d'un unique argument indiscutable en faveur de l'existence de Dieu, est constituée par l'analyse de son nom, cette analyse a été rendue possible par la démarche du Monologion où, à partir de quatre premières preuves, il a été démontré que ce nom est celui de l'Esprit suprême, raison dernière de tout ce qui est, lequel ne fait qu'un avec le Dieu de la foi, découvert dans l'expérience religieuse. Et si, dans le Liber apologeticus, la discussion avec Gaunilon déplace l'argumentation d'Anselme du plan de la réflexion sur la foi vers celui de la théorie de la connaissance et rompt par conséquent avec la démarche du Monologion et du Proslogion, dans le De veritate, il tente de les unifier. Ainsi voit-on que son but, dans presque toute son cuvre, n'était pas tant de prouver que Dieu existe, que de résoudre le problème des rapports de la raison et de la foi, plus exactement de la foi avec la raison.

"Hegel ou la totalité pensante », comme l'indique le titre de la $\mathrm{III}^{\mathrm{e}}$ partie (p. 421), qui demande à être expliqué. Et c'est à quoi s'applique Girard en faisant remarquer que, pour Hegel, la philosophie est la science de l'Absolu conçu comme la totalité de l'existant consciente de soi et existant par soi, c'est-à-dire la science de Dieu, dont il estime que l'existence n'est connue avec certitude que grâce à l'argument ontologique découvert par lui chez Anselme. Mais il reproche à celui-ci de ne pas avoir dépassé l'opposition pensée/être ou sujet/objet, dans laquelle il voit pour sa part une unité dialectique, qu'il caractérise comme « l'auto-mouvement [...] de la totalité pensante » (p. 403). Et, de ce fait, l'argument ontologique, pour lui, ne peut consister dans une analyse conceptuelle de l'idée de Dieu permettant de poser l'affirmation de son existence, mais dans la prise de conscience de l'auto-position de l'Absolu se pensant dans cette conscience même. L'auteur montre ensuite, et c'est là le centre de son exposé, que, pour parvenir à cette connaissance de Dieu, il faut, selon Hegel, tout l'ensemble de la démarche philosophique à travers ses différentes étapes représentées par la Logique, la Philosophie de la nature et celle de l'esprit, la première dévoilant l'auto-constitution de l'idée de Dieu, les deux autres assurant le passage de cette idée au Dieu vivant lui-même. Autrement dit, ce n'est ni 
dans l'art, ni dans la religion, en entendant par là la religion chrétienne, que se réalise ce passage en quoi consiste l'argument ontologique, mais dans la philosophie qui exprime conceptuellement cette religion. Si bien que cet argument, chez lui, tel que l'interprète Girard, ne fait pratiquement qu'un avec toute sa philosophie à l'écoute du christianisme et que, comme chez Anselme, mais d'une tout autre façon, il sert de clef à la question des rapports de la raison et de la foi.

Voilà résumé à très grands traits l'essentiel de l'apport de cette étude, à propos de laquelle on peut exprimer une réserve et un regret. La réserve porte sur le caractère exhaustif que son auteur a donné à son exposé de la pensée d'Anselme et de celle de Hegel, car il s'ensuit que, par moments, le lecteur risque de ne plus voir clairement le lien de cet exposé avec leur conception de l'argument ontologique. Le regret a trait aux références dont l'indication n'est pas unifiée, et qui parfois sont incomplètes ou même manquent.

Jean ÉCOLE

Jean Jolivet, La Théologie d'Abélard. Paris, Cerf, 1997. 12,5 × 19,5, 139 p., bibliogr. (Initiations au Moyen Âge).

Cette publication de La Théologie d'Abélard se veut une introduction simple aux principales cuvres d'Abélard, et notamment à ses trois Théologies. La présentation choisie par Jean Jolivet privilégie une approche œuvre par œuvre et non chronologique ou thématique, ce qui permet de mieux cerner Abélard dans le déploiement de sa pensée.

Mettant de côté ses ouvrages antérieurs, plus techniques et plus érudits, sur Abélard, J. Jolivet commence par présenter son personnage à travers les jugements portés sur lui au long des siècles. Condamnées par saint Bernard, ses vues sur la foi et la Trinité poussent son contemporain Guillaume de Saint-Thierry à parler de lui en termes « d'ennemi intérieur [à] l'Église ". C'est au xIx siècle surtout que la tradition historique fera de lui le héraut médiéval de la raison: Charles de Rémusat et Hauréau le décrivent comme le libérateur de l'esprit humain face aux brumes de l'autorité et de la foi. Les critiques contemporains ont montré l'anachronisme de ces positions.

Chacun des chapitres étant consacré à une œuvre ou à un groupe d'œuvres regroupées selon une unité de genre, Sic et Non, Éthique, commentaires, le premier grand chapitre recouvre les trois textes intitulés Theologia. J. Jolivet fait d'ailleurs remarquer le caractère novateur de ces titres, puisque Abélard est le premier à utiliser ce terme pour désigner ce genre d'œuvres commentant la sacra pagina (p. 1920). Le but de l'auteur est de montrer à la fois le côté novateur d'Abélard, par rapport à Anselme de Cantorbery et à la tradition des gloses et sentences « classiques » des explications de la Bible, ainsi que son insertion dans le xil ${ }^{\mathrm{e}}$ siècle.

Contrairement à une interprétation « rationaliste », J. Jolivet s'emploie à montrer que, pour Abélard aussi, « la foi doit précéder la raison ». Le but de la théologie est 
davantage d'expliquer des formules et leur accord avec la raison que de les expliquer afin de croire. La formule d'Abélard: "On ne peut rien croire sans l'avoir compris ", n'indique donc pas une prééminence de la raison sur la foi qui ne ferait qu'en découler, mais elle signifie bien plutôt le caractère essentiel de la compréhension du sens de ces formules; la foi ne saurait se passer d'une activité de la raison, quand bien même elle la précède. Loin de détruire la foi, la dialectique va permettre de l'assurer plus solidement; cependant, s'il est nécessaire à la foi de s'appuyer sur la dialectique, elle doit aussi la dépasser. C'est sur la question de la Trinité qu'Abélard va pouvoir mettre en cuvre sa méthode logico-sémantique, afin de montrer comment le Dieu unique peut être trois Personnes. Cependant, vouloir rendre accessible à la raison le caractère mystérieux de la Trinité divine lui vaudra d'être condamné une première fois à Soissons.

Un exemple rapide peut permettre de mieux cerner la nouvelle méthode d'Abélard : le premier livre de la Theologia Summi boni utilise la sémantique, afin de montrer que les perfections de Dieu, la Toute-Puissance, la Sagesse et la Bonté sont, en fait, les «propres » de chacune des trois Personnes de la Trinité, bien que cette fonction de propre n'interdise en rien d'attribuer au Fils la puissance. Le " propre " est, ici, le nom particulier que le Bien suprême prend chez chacune des trois Personnes. C'est à une preuve dialectique que le deuxième livre est consacré. Le "propre " n'a plus un sens sémantique, mais énonce la place de chaque Personne dans l'ordre de la procession : le Fils est engendré par le Père et le Saint-Esprit procède à la fois du Père et du Fils. Le deuxième usage de ce terme de "propre " « relève de la logique et plus précisément de l'Isagogê de Porphyre » (p. 31).

Les nombreuses références bibliographiques de J. Jolivet à des commentaires plus érudits lui permettent de passer rapidement sur le problème de l'assimilation de l'Âme du monde platonicienne à l'Esprit-Saint ou sur la critique de Roscelin, accusé par Abélard de trithéisme, par exemple.

C'est donc sur le sens des mots et la possibilité ou non de les attribuer de manière propre et non figurée aux êtres non substantiels que porte la méthode abélardienne. Redressant l'image que l'on peut se faire d'Abélard, J. Jolivet montre bien que celui-ci n'a aucunement pour but la recherche d'une théologie déduite rationnellement; il ne s'agit pas même, pour lui, de retrouver par la raison seule les données de la foi chrétienne, mais, les ayant posées et affirmées, de les exposer sans contradiction avec les règles du trivium. Abélard reprend d'ailleurs la critique de Boèce à l'égard de la raison : les catégories d'Aristote comme les mots de notre langage sont incapables d'exprimer Dieu et sa transcendance.

Des nuances qu'apportent les deux dernières Théologies, nous ne reprendrons que celle qui valut à Abélard sa deuxième condamnation, par saint Bernard au concile de Sens. Reprenant la formule de saint Paul sur la foi comme «argument de choses invisibles », Abélard transforme cet argumentum en un existimatio. Les accusateurs d'Abélard voient en ce terme une reprise des opinions des sceptiques et des dialecticiens, alors qu'Abélard ne semble vouloir insister que sur la radicale séparation entre deux genres de connaissance : celle que nous procurent les sens et la foi.

En ce qui concerne les autres æuvres, on peut simplement insister sur un point de la méthode du Sic et non que J. Jolivet met bien en valeur: la forme de la quaestio n'est pas une méthode novatrice qui serait le signe de l'esprit téméraire et libre d'Abélard. Si le but d'Abélard est "de concilier les autorités" avec « une doctrine 
considérée comme norme de vérité » (citations reprises par J. Jolivet d'un article de R. Bultot), on ne peut plus affirmer qu'Abélard aurait été l'unique porteur du flambeau de la raison dans un siècle obscur.

En ce qui concerne l'Éthique, J. Jolivet insiste, après Marie-Dominique Chenu, sur «l'éveil de la conscience dans la civilisation médiévale », faisant donc porter le poids de son commentaire sur le rôle crucial de l'intention, et non de l'œuvre ou de l'acte, chez Abélard. On peut regretter que la dimension de la collection n'ait pas permis de développer davantage le rapport du sujet et de son destin d'élu et de damné dans la responsabilité du péché, et de replacer ainsi l'Éthique dans un cadre plus théologique.

Ce petit livre permet donc de se faire une idée globale plus juste d'Abélard dans son siècle : J. Jolivet, après de nombreux livres consacrés principalement au " nonréalisme " logique de notre auteur, nous montre la pensée d'Abélard parmi celle de ses contemporains, qu'il les ait lui-même critiqués (ses maîtres de Laon), ou qu'il en ait été la cible (saint Bernard notamment). Abélard n'apparaît donc plus comme une anomalie, mais comme un homme à la fois porteur d'une nouvelle méthode - bien qu'Abélard n'ait jamais réellement étudié que les premiers livres de l'Organon, les Analytiques n'ayant été réintroduits dans le monde latin qu'au milieu du XII ${ }^{e}$ siècle - et d'une foi traditionnelle dans les dogmes chrétiens.

Sylvie RANC-PuECH

François BERRIOT, Spiritualités, hétérodoxies et imaginaires. Études sur le Moyen Âge et la Renaissance. Saint-Étienne, Publications de l'université de SaintÉtienne, 1994. $16 \times 24,420$ p., index (Centre de recherches des langues et de la communication, université de Corse, centre de la Renaissance et de l'Âge classique, université de Saint-Étienne).

Voilà vingt ans, François Berriot soutenait une thèse à l'université de Nice qui se situait au cœur du débat ouvert par Henri Busson et Lucien Febvre sur l'existence de l'athéisme au xvI ${ }^{\mathrm{e}}$ siècle. Son travail, paru en 1976 aux éditions du Cerf sous le titre Athéismes et athéistes au xvi' siècle en France, connaît aujourd'hui une suite sous la forme d'un volume d'articles qui élargit le corpus de réflexion dans le temps et l'espace. Du XIV ${ }^{e}$ au $x{ }^{2} I^{e}$ siècle, de l'orthodoxie chrétienne, de ses déviations, à l'islam, F. Berriot nous offre une série d'études fort intéressantes sur la manière dont est modalisée la spiritualité (par l'intermédiaire des vies de saints, des disciplines conventuelles, des cérémonies cultuelles), vécue par les fỉdèles ou rejetée par des formes souvent complexes et fascinantes d'impiété. Ce volume d'articles poursuit le même enjeu que le travail qu'avait publié le Cerf : montrer l'extrême diversité des modèles et des comportements orthodoxes ou déviants, organiser - grâce aux multiples éclairages et aux cas cités - un faisceau de points de vue qui ébranle les idées reçues et les habitudes mentales.

D'une Vie de sainte Claire traduite en français au XIv siècle, à la romanisation de la liturgie, des lectures symboliques de la Tapisserie Crestienne de Jean Germain 
(1457) à l'examen d'un livre d'Heures du $x v^{e}$ siècle, F. Berriot suit le parcours de la pédagogie religieuse quand elle doit en même temps se faire modèle et accepter de croiser une spiritualité populaire et des habitudes mentales propres au Moyen Âge. Ce premier parcours s'achève sur l'étude d'un manuscrit d'Entretiens spirituels du $X V I I{ }^{e}$ siècle où l'on suit l'apologétique quand elle rencontre le quiétisme et le libertinage, sans renoncer - comme on aurait parfois trop tendance à le croire - aux merveilles d'un monde que la raison n'a pas encore désenchantée. La deuxième partie de son livre aborde la manière qu'on avait de se représenter le monde arabe. Cette section tout à fait passionnante parcourt ce champ depuis le rôle joué par la chanson de geste qui imprègne l'imaginaire occidental, élabore le mythe d'un Saladin preux et occidentalisé, jusqu'aux réflexions d'un Jean Bodin, quand il aborde, dans son incroyable Colloquium heptaplomeres la question de la religion naturelle. Des images rassurantes ou répulsives de l'islam au rêve polémique d'un monothéisme pur, cette section débouche sur le problème de la liberté de conscience à la Renaissance. C'est ce dernier point qui articule le passage aux différentes figures de l'hétérodoxie que F. Berriot aborde alors, dans une troisième partie, retrouvant des individualités aussi étonnantes que celles de Gautier Le Leu, Haymon de La Fosse, Noël Journet ou Jacques Gruet quand se mêlent, au carrefour de l'impiété, libertinage de mœurs, opposition politique et révolte intellectuelle. La dernière partie de l'ouvrage, consacrée aux clés des songes médiévales, aux dormeurs de la Renaissance, se clôt sur le non-dit de l'ensemble du livre : l'imaginaire, le jeu symbolique de la mort et de la résurrection rêvées, le deuil enfin sont non seulement au cour de l'entreprise religieuse, de ses modèles, de ses élaborations doctrinales, mais aussi de l'attitude des croyants, comme des impies.

L'ouvrage de F. Berriot vaut ainsi surtout pour la richesse des textes qu'il nous offre, l'incroyable diversité des attitudes et des questions qui nous sont présentées. Les recherches en cours sur la spiritualité (sur ses modèles, ses acteurs et ses détracteurs) ne pourront que s'enrichir de ce type d'ouvrage qui ouvre des pistes et des champs de réflexion, et nous invite à écouter et faire parler les vies singulières et fascinantes des saints et des impies.

Sophie HouDARD

Silvana Seidel Menchi, Érasme hérétique. Réforme et inquisition dans l'Italie du xvf siècle. Trad. de l'italien par Pierre-Antoine Fabre. Paris, Seuil/Gallimard, 1996. $15,3 \times 24,446$ p., bibliogr., index (Hautes études).

Peu de livres récents donnent un aperçu aussi vivant de l'effervescence religieuse du Xvi siècle que celui de Silvana Seidel Menchi, si heureusement traduit. Outre le talent de l'auteur, ce fait est imputable aux sources qu'elle utilise, ces innombrables procès inquisitoriaux qu'elle a patiemment collationnés là où ils sont disponibles. Ces textes sont remarquables par leur précision, leur spontanéité, la pénétration psychologique d'inquisiteurs habitués à fouiller les consciences. Le nombre impressionnant d'exemples que peut présenter l'auteur permet d'éviter les inconvénients d'une « micro-histoire » trop souvent séduite par l'exceptionnel. 
La thèse développée par S. Seidel Menchi à partir de ces procès est la suivante : le véritable inspirateur du mouvement hérétique italien est Érasme. Dès les années 1520, malgré les sympathies que le maître de Rotterdam peut susciter en Curie, parfois même auprès des papes, le milieu conservateur italien tire à boulets rouges sur l'humaniste, dont Luther n'aurait fait que suivre les leçons. Cette précocité et cette ampleur du rejet d'Érasme comme hérétique font toute l'originalité italienne, notamment par rapport à l'Espagne où il sera beaucoup plus tardif. Mais en même temps, toute une génération de jeunes lettrés succombe à la séduction du style et des idées du Batave. C'est elle qui sera la victime de la phase la plus dure de la répression inquisitoriale dans les années 1550 et 1560 .

Érasme luthérien? Certes, entre la théologie de Luther et les idées d'Érasme existe une ligne nette de rupture, qui s'appelle le libre arbitre. Érasme a toujours refusé de rejoindre la dissidence religieuse, même si son appui à l'ancienne Église est bien ambigu. Il reste entre les deux Réformes, et $\mathrm{S}$. Seidel Menchi voit dans les idées érasmiennes " le pont jeté vers l'autre rive, dans une étape transitoire de l'itinéraire religieux » (p. 90). Mais, comme elle l'ajoute, cela n'est vrai que de la diaspora des dissidents italiens, contraints par l'exil d'adopter les orthodoxies luthériennes ou calvinistes. Pour ceux qui restèrent et finirent victimes de l'Inquisition, Érasme n'avait rien d'une simple étape. Et la dissidence intérieure italienne garda ainsi cette indifférence typiquement érasmienne à l'égard des définitions dogmatiques trop contraignantes, qu'elles viennent de Trente ou de Genève, cette aspiration à une religion plus intérieure, ce goût pour la liberté spirituelle.

L'influence d'Érasme s'exerce de façon prépondérante sur le milieu hérétique italien tout simplement parce qu'au contraire de la France ou des Pays-Bas, il ne pourra jamais s'organiser en une véritable Église clandestine. Ainsi, on peut expliquer les traits originaux de la dissidence religieuse dans la péninsule, qui contrastent tant avec les théologies protestantes du nord des Alpes : une confiance dans la miséricorde divine qui, contrairement à Luther, puise sa source dans une vision optimiste de la nature humaine; une certitude que le Ciel est ouvert à tous et non restreint à un petit nombre d'élus, comme les frères ennemis des deux Réformes le martèlent. L'influence d'Érasme se retrouve aussi dans l'éloge du mariage chrétien, repris cette fois-ci par les Réformateurs d'outre-Alpes.

Les dizaines de témoins que $S$. Seidel Menchi peut produire à l'appui de sa thèse ne citent cependant pas tous explicitement le maître de Rotterdam comme leur inspirateur. On voit là une des limites du livre, qui attribue peut-être un peu trop systématiquement à une influence érasmienne des propos dont l'origine est sans doute plus banale. Les critiques contre le jeûne, les voux monastiques, le culte des saints, les images peuvent tout aussi bien venir d'un vieux fond anticlérical, qui n'avait pas attendu Érasme pour se manifester. Ce fond est le bien commun de laïcs cultivés maîtres d'école, notaires, artisans alphabétisés qui sont les victimes privilégiées de l'appareil inquisitorial. Ainsi s'impose tragiquement dans la péninsule un conformisme intellectuel qui bannit l'œuvre d'Érasme des bibliothèques italiennes, sinon dans des versions fades et expurgées. Ce succès de l'Inquisition s'explique-t-il seulement par la répression? Si tout le livre semble conduire à cette conclusion, le dernier cas cité, celui d'un fileur vénitien exécuté en juillet 1587, esquisse une autre piste. Ses convictions érasmiennes étaient devenues anachroniques dans une Italie gagnée - et pas seulement par la force - à la Contre-Réforme. 
Bien des aspects de ce beau livre mériteraient un développement plus ample : sa périodisation très fine des âges inquisitoriaux, sa présentation du doute érasmien, le lien entre parti français et dissidence religieuse en Italie, etc. Leur simple mention engagera à la lecture d'un ouvrage dont la richesse ne se résume pas.

Alain TALLON

Frank LESTRINGANT, Une sainte horreur ou le voyage en eucharistie, $x v f^{\prime}$-xvII siècle. Préf. de Pierre Chaunu. Paris, Presses universitaires de France, 1996, 15 ×21,6, $x x-361$ p., bibliogr., index (Histoires).

Ce livre commence et finit avec l'ironie plus ou moins grinçante de Swift: en ouverture, un conte sur trois frères, Peter, Martin et Jack, où le premier, mégalomane et trompeur (le pape), veut faire croire aux deux autres (Luther et Calvin) que du pain est en fait de l'épaule de mouton; en épilogue, un essai qui se veut drôle en proposant comme solution au problème démographique irlandais de manger les enfants d'un an. Eucharistie catholique et anthropophagie : tel est le thème central de ce livre, qui étudie la « Sainte Horreur " protestante - et plus précisément calviniste - devant l'hostie catholique. Horreur sacrée qui aurait fait dire au jeune Agrippa d'Aubigné, sommé de choisir entre le bûcher et la messe, « que l'horreur de la Messe lui ôtait celle du feu ". L'originalité de Frank Lestringant est d'aborder cette horreur armé de sa compétence de linguiste et de sa profonde connaissance de la littérature protestante sur le «sauvage ». De l'anthropophage que les Européens découvrent en Amérique au théophage catholique que combattent les huguenots dans le Vieux Monde, la comparaison s'établit tout naturellement. En l'étudiant "dans ce qui serait un essai de critique charnelle », l'auteur veut donner forme à « l'imaginaire corporel en ceuvre dans le refus protestant de l'Eucharistie catholique » (p. 5).

Dès les débuts de la Réforme, au moins dans l'espace français, cette sainte horreur se manifeste avec violence. Les célèbres placards contre la messe, rédigés par le pasteur Antoine Marcourt et apposés dans la nuit du 17 au 18 octobre 1534, signent irrémédiablement la rupture entre l'Église établie et les dissidents. Rupture due avant tout à la violence du rejet protestant, dont nous imaginons mal l'ampleur aujourd'hui. Ce dégoût viscéral devant le sacrifice sanglant de la messe n'est, comme le souligne justement $F$. Lestringant, « non pas tant une victoire de la raison que le renforcement du tabou religieux » (p. 14). Toucher, manger Dieu, le digérer : voilà qui obsède l'imaginaire réformé. La polémique huguenote traite volontiers du thème, de façon satirique ou horrifiée, souvent scatologique, toujours sanglante. La théologie calviniste oppose au matérialisme catholique une « sémiologie de la disjonction " (p. 22). Le pain signifie l'absence matérielle du Christ autant que sa présence spirituelle. La répugnance devant le sacrifice sanglant devient un des traits de cet imaginaire protestant. L'auteur l'étudie à travers deux cuvres, Hamlet, qu'il restitue à l'univers réformé sur la trace de Stephen Greenblatt, et Les Tragiques, où l'obsession cannibale a une claire fonction de controverse.

Dans sa seconde partie « Tristes tropistes », F. Lestringant reprend le thème, déjà largement évoqué dans ses autres travaux, de la confrontation entre cannibales du 
Nouveau Monde et controverse eucharistique. La courte aventure de la «France australe » voit les huguenots préférer encore la compagnie des sauvages anthropophages à celle de leurs frères ennemis catholiques ou catholicisants. L'auteur avait déjà livré une édition critique du récit fait par Jean de Léry, un des protagonistes calvinistes de cette guerre de religion sous les tropiques. L'écho en Europe est grand, notamment grâce au goût pour la polémique du chef de l'expédition, Villegagnon, ex-protestant revenu à l'eucharistie papiste. Les pamphlétaires genevois lui répondent en l'identifiant au Cyclope cannibale :

« Ce Cyclops inhumain de la celeste place

Faict venir Jésuschrist, pour aux dents le briser,

Et le mange tout vif, pour sa faim appaiser » (cité p. 95).

À côté de ces monstres, le véritable anthropophage soutient bien la comparaison. Sa perversion semble plus naturelle que la volonté catholique de transformer le cuit (pain) en cru (chair du Christ), mutation qui va à rebours de la civilisation. Les jésuites portugais en retour dénoncent "l'ensauvagement" des colons huguenots. L'insistance sur les cruautés commises par les calvinistes en France ou dans les Pays-Bas reprend cette idée de sauvagerie bestiale indissociablement liée à l'hérésie. Dans une surenchère, hélas bien souvent fondée sur des faits réels, les huguenots ne manquent pas de cadavres à opposer à leurs accusateurs catholiques. La légende noire de la conquête de l'Amérique par les Espagnols est même mobilisée. Les gravures reproduites en abondance dans le livre donne une idée de l'obsession macabre qui règne dans cette polémique, obsession de la chair encore fraîche que F. Lestringant rattache avec finesse à la présence réelle du Corps du Christ dans l'hostie.

$\mathrm{Si}$, avec un Montaigne, le débat commence à se rationaliser, Port-Royal le situe pleinement sur le seul terrain de la logique. Mais l'horreur protestante demeure et s'exprime dans un genre nouveau, que F. Lestringant qualifie de «Voyages en Eucharistie », sorte de sous-catégorie d'utopie qui « fait apparaître, sous les traits accusés d'une société théocratique et anthropophage, un condensé de l'horreur qu'éprouvent le protestant et, dans son sillage, le libre penseur pour une violence archaïque indéfiniment resurgie dans le dogme d'un ennemi diabolisé » (p. 293). La lune de Cyrano, les Terres australes, Formose ou l'Irlande deviennent prétexte à cette dénonciation lancinante de la barbarie catholique. Le débat dépasse alors la seule question eucharistique. Au terme de ce beau livre, dense et parfois déroutant, l'auteur en conclut que ce dégoût révèle un véritable malaise de civilisation, un refus de la chair, du sang, du sacrifice qui entérine l'éloignement du sacré.

Alain TALLON

David EL KENZ, Les Bûchers du roi. La culture protestante des martyrs (15231572). Seyssel, Champ Vallon, 1997. 15,5 × 24, 281 p., bibliogr., index (Époques).

S'appuyant principalement sur le martyrologe rédigé par Jean Crespin et sur les gravures illustrant les supplices des hérétiques dans la France de François ${ }^{\text {er }}$ et de 
Henri II, David El Kenz nous livre un bel ouvrage d'anthropologie historique qui veut « redécouvrir cette culture martyrologique de la première communauté calviniste française " (p. 11). Dès ses premiers balbutiements, la Réforme française doit faire face à une répression royale qui n'éprouve plus aucune hésitation après 1534 . Le roi très chrétien a juré lors de son sacre de préserver le royaume de l'hérésiepromesse que Henri II omet très curieusement en 1547. Le dissident est coupable de lèse-majesté divine et humaine et seule la purification par le feu libère la communauté d'une souillure largement assimilable à celle de la sorcellerie. Hérétiques comme sorciers, inspirés par le Malin, bouleversent tous deux l'ordre divinement établi. Confondus dans la même exécration, ils sont victimes de la même logique d'extermination. Le roi chevalier comme son fils sont convaincus de la nécessité comme de la légitimité de la répression qui atteint des sommets, au moins à Paris, dans la seconde moitié des années 1540 , pic que signale l'auteur (p. 130) sans vraiment l'expliquer.

Face à ce pouvoir persécuteur, comment réagissent les autorités de l'Église naissante, à commencer par celui dont le magistère s'impose progressivement aux fidèles français dans les années 1540 , Jean Calvin? La théologie réformée connaît un certain nombre de difficultés à intégrer le martyre parmi ses catégories. Calvin répugne à utiliser le terme. Son respect de l'autorité séculière lui interdit d'y voir un geste de révolte. Il faut accepter la persécution, sans pour autant renoncer à sa foi en dissimulant comme les nicodémites honnis par Calvin, mais aussi sans se laisser aller à une résistance violente. L'autre danger est de tomber dans une interprétation du martyre comme méritoire, sur le modèle catholique. La victime imite certes son Sauveur, mais « éprouver les douleurs du Christ n'est pas un gage méritoire. Ce n'est qu'une consolation face aux troubles rencontrés par le fidèle » (p. 84). La certitude de l'élection vient bien plus de la fidélité témoignée à Dieu que de l'épreuve elle-même. Après la mort, conserver la mémoire du martyr ne doit surtout pas dégénérer en une quelconque vénération. Elle a seulement vocation pédagogique pour confirmer dans la foi ou convertir. L'analyse sociologique très fine des martyrs présents dans Crespin montre quel public est visé : « les couches inférieures des élites citadines » parmi lesquelles l'auteur choisit ses exemples avec prédilection.

Mais ce modèle pédagogique s'effrite dès les années 1550. Malgré les exhortations de Calvin, le martyre réformé devient geste de défi à l'absolutisme persécuteur. La figure emblématique de cette opposition ouverte au pouvoir est celle d'Anne Du Bourg, reprochant la répression à Henri II en plein Parlement. Emprisonné, il est exécuté après la mort du roi, mort interprétée en milieu réformé comme une vengeance divine. Alors même que le martyre prend une signification politique de plus en plus évidente, les catholiques savent réagir devant la sympathie et la pitié provoquées par tant de morts. Ils reprennent la vieille théologie augustinienne des martyrs du Diable : l'hérétique peut bien mourir pour sa foi diabolique; cela n'est pas une preuve de sa validité, bien au contraire. Après 1562, le parti catholique sait aussi promouvoir ses propres martyrs, prêtres, religieux, mais aussi François de Guise canonisé par la propagande catholique après son assassinat.

La martyrologie réformée donne l'impression de décliner après 1562 pour laisser la place au récit du massacre anonyme. En 1572, seul Coligny, figure au confluent de l'idéologie nobiliaire et de la piété huguenote, acquiert une véritable stature de martyr. La Réforme sécularise jusqu'à ses souffrances, sans aucun doute pour mieux 
préserver la seule gloire de Dieu. Le premier bénéficiaire en sera l'État royal, qui ne se verra plus opposé la légitimité sacrée du martyr. Son effacement rend possible la pacification henricienne.

Alain TALLON

Abraham von Franckenberg, Briefwechsel. Eingel. und hrsg. von Joachim Telle. Stuttgart, Bad Cannstatt, Frommann-Holzboog, 1995. $18 \times 24,5,437$ p., bibliogr., index.

Abraham von Franckenberg (1595-1652), contemporain des désastres de la guerre de Trente Ans, fait partie d'une génération de penseurs et d'écrivains silésiens à laquelle le lecteur français a été introduit, entre autres, par le livre de Bernard Gorceix (Flambée et agonie, Sisteron, Éd. Présence, 1977) consacré aux grandes figures du renouveau mystique en Basse-Silésie au xviI siècle : Tschesch, Czepko..., et dont il connaît les origines par les travaux d'Alexandre Koyré, le livre sur Jakob Böhme et les articles repris dans le volume Mystiques, spirituels, alchimistes du xve siècle allemand (Paris, Gallimard, 1971). Le nom de Franckenberg est surtout attaché à son activité de biographe et d'éditeur de Böhme, et secondairement à des traités de mystique et de philosophie de la nature (voir Siegfried Wollgast, Philosophie in Deutschland zwischen Reformation und Aufklärung 1550-1650, Berlin, AkademieVerlag, 1988). Bien qu'il ait passé l'essentiel de sa vie, après son «illuminatio » qui le fit renoncer aux études, retiré sur les terres du domaine familial, Franckenberg a entretenu une correspondance active, dont la liste trace le contour d'un réseau qui relie la Silésie à Vienne et Amsterdam (voir sa lettre au célèbre rabbin Menasseh ben Israël).

L'édition d'une partie de ses lettres ne change pas essentiellement l'image que l'on avait de l'homme, mais déplace certains accents : l'écrivain et le poète reculent au profit de l'érudit, du directeur de conscience et du philosophe de la nature - on connaît les contacts de Franckenberg avec des médecins et des milieux alchimistes, comme en témoigne le catalogue de sa bibliothèque, établi par Janos Bruckner. (Abraham von Franckenberg. A bibliographical catalogue with a short list of his library, Wiesbaden, Harrassowitz (Beiträge zum Buch \& Bibliothekswesen, 25), 1988). Surtout, elle le fait apparaître comme représentant de la piété de son temps: il n'est pas un simple porte-parole de Böhme, comme on l'a dit parfois, et d'ailleurs, ce n'est pas comme böhmien qu'il a été perçu et vilipendé par les théologiens orthodoxes, mais plutôt comme «Fanatiker » disciple de Valentin Weigel. D'autres influences sont sensibles, et sans doute plus décisives dans son itinéraire spirituel : Johannes Tauler, Thomas a Kempis, Johann Arndt, la Theologia Deutsch.

Même les détracteurs de Franckenberg, qui lui reprochent son fanatisme et son indifférentisme, saluent sa piété authentique, telle qu'elle se manifeste dans de nombreuses lettres d'édification ou de consolation spirituelle. Dans le prisme de sa correspondance, on reconnaît les traits de la crise religieuse qui ébranle les contemporains de la guerre de Trente Ans. Comment trouver le chemin de la foi 
dans une époque où toutes lois semblent renversées et la nature humaine irrémédiablement pervertie?

Franckenberg, qui se rattache à une mouvance du luthéranisme dissident, défend des positions qui annoncent celles du piétisme radical. On reconnaîtra, classiquement, les thèmes de la polémique contre les institutions religieuses et universitaires (les " arguties de la raison "), de l'ébranlement des autorités (la théologie orthodoxe, la philosophie aristotélicienne, la médecine de Galien) et du recentrement sur les facultés de l'âme. Sans rejeter entièrement le programme de formation humaniste, Franckenberg met l'accent sur la réforme intérieure. La vie spirituelle est sous le signe de la relativisation des querelles confessionnelles. La condamnation des sectaires (voir en particulier les passages sur les sociniens), le refus du « chiliasme violent " au profit du "chiliasme subtil " (l'humanité ne vit pas encore dans le temps du Jugement, mais dans celui des Évangiles), l'ouverture à certaines traditions spirituelles du judaïsme, sont la réponse pacifique de dissidents protestants à la crise de la piété et aux maux de l'Église luthérienne.

Par l'éclairage qu'il apporte, cet ouvrage s'inscrit dans la dynamique des recherches actuelles sur le piétisme allemand, soucieuses de mieux cerner les contours du mouvement en l'inscrivant dans l'horizon de l'appel à la réforme religieuse, pour en faire mieux ressortir l'originalité propre.

Anne LAGNY

Denise Leduc-Fayette, Fénelon et l'amour de Dieu. Paris, Presses universitaires de France, 1996. 11,5 × 17,5, 128 p., bibliogr. (Philosophies, 66).

Cet ouvrage très éclairant restitue au plus près la tentative systématique de Fénelon, qui entreprend de traduire dans le registre conceptuel l'expérience intime du pur amour de Dieu. Les très nombreuses références utilisées par l'auteur ne peuvent être mentionnées ici, et nous nous contenterons de reprendre la thèse qui nous semble éclairer l'ensemble des développements proposés dans ce livre : le travail de Denise Leduc-Fayette permet de lire, dans le pur amour fénelonien, l'expérience d'une possibilité qui, au-delà des interprétations modales, doit délivrer l'homme du souci de l'effectivité mondaine, pour lui manifester sa véritable destination.

Dans un premier chapitre, D. Leduc-Fayette dresse un tableau très utile de Fénelon dans son temps, qui met en évidence la façon dont l'archevêque de Cambrai prend acte des principales positions théologiques de l'époque, dans la constitution de sa propre pensée. L'Examen de la théologie mystique de Chéron (1657) marque un certain déclin du mysticisme, auquel on oppose désormais l'affirmation selon laquelle il n'est pas d'amour sans connaissance. Si Fénelon ne peut entièrement souscrire à cette thèse, nous verrons qu'il ne manque pas, cependant, de laisser sa place à la raison, dans l'amour de Dieu - l'usage complexe, mais parfaitement justifié, de cette faculté constitue d'ailleurs un des grands enjeux de sa pensée. Corrélativement, Fénelon s'oppose aux jansénistes parce qu'il défend la grâce congrue; dans cette perspective, il distingue la prédestination de la prescience divine. Et il 
enregistre les conclusions de la sixième session du concile de Trente, qui insiste sur la nécessité de coopérer à la grâce; c'est pourquoi il ne peut adopter les positions de Miguel de Molinos, qui prônait une totale résignation à la volonté de Dieu. En somme, les positions théologiques adoptées ou réfutées par Fénelon lui permettent de passer au crible l'expérience mystique, pour conférer à celle-ci l'orthodoxie qui semble toujours lui faire défaut. Cette entreprise est orientée par une grande distinction, sur laquelle D. Leduc-Fayette apporte toutes les précisions requises : celle qui sépare une passivité inacceptable en milieu catholique, de la « passiveté » qui enveloppe notre coopération. Sous ce point de vue, l'amour de Dieu commande une certaine disposition de notre âme à l'activité, qui justifie à quelque égard l'intervention de la raison.

On comprend alors qu'une expression systématique du pur amour trouve sa place dans la théologie, même si elle ne peut donner lieu à des formulations conceptuelles irrévocables. Quelles raisons autorisent donc Fénelon à parler d'un « système " de l'amour de Dieu? Trois motifs apparaissent très clairement, dans le deuxième chapitre : la théologie mystique s'organise selon un principe unique; il est légitime d'essayer de la traduire en concepts, puisqu'elle réclame la participation de l'homme raisonnable; Fénelon soutient, contre Molinos, que le pur amour est, en droit, accessible à tous - cette expérience, éminemment individuelle, possède donc une valeur générale. Ce dernier point nous paraît très important, pourvu qu'on lui restitue toute sa portée - il signifie bien que l'amour de Dieu est, pour l'homme, chose possible, qu'il constitue même la plus haute possibilité. Cette thèse oriente discrètement mais efficacement tout le travail de $\mathrm{D}$. Leduc-Fayette. Avant de développer ce point, il faut évidemment l'assortir de deux restrictions importantes, qui engagent le statut et les prétentions du système. D'une part, il est impossible de déterminer une fois pour toutes cette possibilité selon l'enchaînement discursif des concepts, puisque l'infinité intensive de Dieu demande une adhésion directe qui, comme il sera montré plus tard, requiert une totale spontanéité. D'autre part, la place de la philosophie dans la constitution du système du pur amour reste subordonnée à une inspiration religieuse qui s'accomplira dans la prière.

L'amour de Dieu requiert, comme chacun sait, une désappropriation de l'ego, dont le troisième chapitre du livre s'emploie à distinguer les caractères. L'allure cartésienne des formulations de Fénelon ne doit donc pas cacher qu'il subvertit les positions de Descartes lui-même. Peut-être faut-il insister ici, cependant, sur le fait que cette torsion ne s'effectue pas essentiellement sur la question de la subordination de l'ego à l'égard de Dieu, que les Méditations elles-mêmes établissent. On insistera davantage sur le principe d'un déplacement dont $D$. Leduc-Fayette, par ailleurs, enregistre très bien les manifestations frappantes. Puisque l'âme sort d'ellemême, dans l'expérience fénelonienne du pur amour, elle accède à des considérations qui restaient interdites à l'ego cartésien. Celui-ci ne pouvait jamais connaître Dieu que dans la mise en ouvre d'une causalité efficiente, et n'avait pas les moyens internes de se prononcer sur les causes finales. En revanche, dans l'épreuve mystique, l'homme fait l'expérience d'un excès qui le conduit à éprouver l'insuffisance d'une description simplement mécaniste de la nature. L'ordre même mis en évidence chez Malebranche restreint la manifestation de Dieu dans les phénomènes, selon Fénelon : l'harmonie de l'univers doit avant tout suggérer l'infinité du dessein de Dieu. Insistons bien sur le fait que, selon une perspective cartésienne, cette consi- 
dération des fins implique que l'ego sorte de lui-même - bien plus, l'argument cosmologique ne vaut que sous l'horizon de cette désappropriation du moi. Fénelon donne finalement raison à Descartes, mais il l'assortit d'une perspective théologique : il demeure exact que je ne puis vraiment savoir que Dieu veut se communiquer à moi, si je reste enfermé dans les bornes qui me sont naturellement assignées; mais comme la réalité de cette communication est attestée par la révélation, je dois impérativement renoncer aux perspectives simplement humaines. La possibilité de l'amour de Dieu ne fonctionnera donc pas selon des moyens finis. Tandis que l'individu reconnaît spontanément (c'est la voie la plus simple) l'harmonie dans l'univers, il ne peut vraiment certifier cette beauté qu'en abandonnant ce point de vue encore impur. L'important est alors, pour chacun, de reconnaître sa destination dans son origine contingente et finie, et de restituer en lui-même la splendeur du projet divin. Parce que le sujet de Fénelon est encore cartésien, il doit renoncer à luimême pour adhérer à la volonté finale de Dieu - on conçoit alors le sens que Fénelon donne à la thèse de la création des vérités éternelles : elle manifeste la toutepuissance de Dieu, mais elle doit encore montrer que cette puissance est orientée. Quoi qu'il en soit, la conception théologique de Fénelon (qui, on le voit, est aussi gnoséologique) lui interdit évidemment de ratifier la distinction malebranchiste entre un amour de soi bien légitime et un amour propre condamnable. C'est qu'il ne s'agit pas simplement de renoncer aux intérêts empiriques (ce que tout le monde accorde) : il faut abandonner toute perspective subjective, qui ne me permet pas d'accéder à la volonté divine - de la comprendre et de m'y conformer. L'amour que nous portons à Dieu ne doit être que celui qu'il veut se porter au travers de notre moindre degré d'être. Le problème de l'espérance se pose donc dès à présent, mais en termes philosophiques : la désappropriation de l'ego est requise pour que s'opère l'identification $d u$ Dieu, et au Dieu qui nous est promis. En somme, l'ego fénelonien ne se conçoit pas dans les seuls termes de l'examen modal.

C'est pourquoi l'ouvrage se poursuit par l'étude de la structure extatique de l'amour (chap. Iv). La seule réalisation de mon essence conforme au projet divin réside dans l'inscription de l'amour de Dieu en mon âme, qui provoque une « union

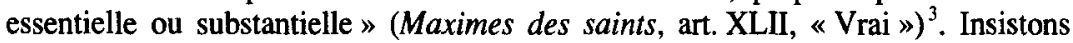
encore une fois sur la possibilité de cette union : puisqu'il s'agit de restituer sa place à la volonté divine, je puis aimer Dieu dès lors seulement que je le veux, autrement dit, dès que je veux qu'il veuille en moi. Nous reviendrons plus tard sur la condition négative de cette substitution, qui nous paraît capitale; mais signalons dès à présent qu'à cette fin l'homme doit ici renoncer à l'illusion de son effectivité propre. Selon cette exigence, il est clair que le recentrement authentique que produit l'extase (je trouve ma place dans celle que je restitue à Dieu) implique d'abord que je sorte du domaine étroit de ce que je puis posséder — en un sens large, où la possession désigne tout ce que j'imagine relever de mon action propre: "Tout nombre est bientôt épuisé; toute composition a des bornes étroites; tout ce qui est plus d'un, est infiniment moins qu'un $»$ (Traité de l'existence de Dieu, partie II, chap. v, art. II).

3. Tous les textes mentionnés sont de FÉNELON : on consultera principalement l'ensemble de ses œuvres dans l'édition de Versailles (Gosselin et Caron), 1820-1830, 35 vol., et l'édition scientifique actuellement en cours, dir. par Jacques LE BRUN, Paris, Gallimard (Bibliothèque de la Pléiade), vol. I, 1983. 
Ainsi la mort de l'effectivité humaine, des projets individuels inscrits dans le temps factice de l'histoire, se traduit-elle positivement par ce que D. Leduc-Fayette nomme justement «l'actualité ponctuelle de l'extase ». Comprenons qu'il n'est pas question ici de se résigner, comme on se résigne justement aux événements empiriques, mais plutôt de se soustraire à l'enchevêtrement des désirs qui agitent le thêâtre des hommes - c'est la première condition de l'état passif d'indifférence, qui fait l'objet du chapitre $v$ du livre.

Le sens de l'indifférence réside ainsi dans l'annulation de tous les objets qui fascinent la volonté ordinaire. Il faut enregistrer ici une précision très éclairante apportée par D. Leduc-Fayette, dans ce chapitre $v$ : l'amour pur ne consiste peut-être même pas dans le fait de vouloir ce que Dieu veut (que puis-je en savoir, sans y mêler mes propres motifs?), mais il nous conduit à « vouloir de ce qu'il veut ». L'amour pur dessine une possibilité pure, celle de la présence de l'être en moi, abstraction faite des figures de l'effectivité sous lesquelles nous le restreignons aux dimensions humaines : «[...] ne faut compter pour rien ce qui se fait [...], pour s'unir à ce qui est, c'est-à-dire l'être parfait et immuable » (Opuscules spirituels, I, XIII). Tel est finalement le sens de la fameuse supposition impossible, selon laquelle l'âme voudrait souffrir toutes les peines de l'enfer, si telle était la volonté de Dieu : la supposition impossible est un écart par lequel nous éprouvons vraiment la mort, en notre âme, de la nostalgie d'une effectivité humaine. L'indifférence ne désigne donc pas une morne cessation d'action, mais l'action de Dieu qui conduit notre volonté : «La détermination absolue à ne rien vouloir ne serait plus le désintéressement, mais l'extinction de l'amour, qui est un désir et une volonté véritable » (Maximes des saints, art. V, « Vrai »). L'amour de Dieu est possible, puisqu'il nous appartient de nous déterminer à laisser Dieu vouloir en nous : mais il faut alors tenir que le destin de la volonté humaine est de s'anéantir - la plus haute volition dont nous sommes susceptibles doit abolir le mode d'effectuation quotidien de notre volonté.

Les longues conclusions rassemblées par D. Leduc-Fayette présentent le plus haut intérêt pour la compréhension générale de l'œuvre de Fénelon. L'auteur mentionne d'abord la prétention de l'archevêque de Cambrai à établir une authentique philosophie naturelle. Simplement, nous devons renoncer à l'illusion de tout comprendre, et tenter de reconnaître le principe par lequel tout peut être. Sous ce point de vue, ce n'est pas le pur amour qui détruit l'espérance, mais bien l'entreprise des savants qui, quand ils désirent tout voir, en «extensivité », s'exposent à un échec proprement désespérant. L'identification du principe de toutes choses, par suite, doit s'accomplir dans la prière. Fénelon n'est jamais misologue (voir à ce sujet l'étude de Jacqueline Lagrée, "Quiétude et inquiétude de la raison ", in Fénelon. Philosophie et spiritualité, textes réunis par D. Leduc-Fayette, Paris, Droz, 1996) - il ne prône pas un scepticisme qui s'abîmerait dans le constat des contradictions incessantes de l'humaine raison, mais il veut conduire la raison jusqu'à ses limites, pour montrer qu'ensuite, l'homme doit prier. La défaillance définit, pour ainsi dire, la pointe de la raison. Aussi bien la nécessité de la prière est-elle encore philosophique : la foi est le seul mode sous lequel nous pouvons certifier le projet divin, jusque dans la nature. L'ouvrage envisage enfin le problème traditionnel de l'espérance. On ne peut oublier que, sous le jour d'une raison finalisée par la prière, cette vertu théologale appartient nécessairement à la contemplation de Dieu. Comme 
l'écrit D. Leduc-Fayette : «Dieu n'est pas exclusivement créateur, il est Providence. " Entendons par là que l'amour de Dieu ne peut se satisfaire des seules considérations modales, accessibles à la raison, mais qu'il porte sur le Dieu que nous espérons, que nous attendons vraiment. Tel est, finalement, le moteur de la conversion chez Fénelon : non plus la libido qui agite les sciences, mais l'attente qui nous dispose à recevoir de Dieu un mouvement adéquat. Cette attente doit reconduire en sa pureté l'instant de l'extase, qui seul dispose un être changeant à la présence divine, au lieu qu'une espérance déterminée consiste en fin de compte à projeter des bornes : « Nous sommes nourris comme Jésus-Christ de la volonté de son Père, que la Providence nous apporte dans le moment présent » (Lettres spirituelles, $\mathrm{n}^{\circ}$ 187). L'homme doit faire abstraction de la vertu d'espérance, afin de s'abandonner sans réserve à l'amour de Dieu - la supposition impossible fonde et éprouve cette possibilité, pour l'âme, de se libérer vraiment des bornes qui affectent l'homme en ce monde. À l'extrême, elle fortifie une espérance authentique, qui ne peut se loger que dans un désintéressement absolu, pour n'être pas indigne de la Providence et se disposer à tout recevoir. Fénelon montre ainsi qu'une philosophie de l'amour de Dieu définit la seule expérience humaine d'une possibilité dégagée de toute effectivité, ou limitation.

André Charrak

De l'Examen de la religion attribuable à Jean Lévesque de Burigny. Éd. critique par Sergio LaNDuCCI, suivie d'un appendice documentaire. Paris/Oxford, Universitas/Voltaire Foundation, 1996. 17,5 × 24, 148 p., bibliogr., index (Libre pensée et littérature clandestine, 4).

L'un des principaux intérêts de ce texte, qui examine les justifications de la religion chrétienne, en critiquant in fine l'idée de révélation surnaturelle, réside dans son caractère de transition : dépendant encore de la formulation des questions telle qu'elle avait été posée dans les controverses entre catholiques et protestants de la fin du $x v I^{e}$ siècle, il ouvre la voie au débat sur le déisme et la religion naturelle qui occupera la période des Lumières.

Dans son introduction, Sergio Landucci en resitue très clairement le contexte et l'enjeu. Il reprend en particulier l'hypothèse selon laquelle ce texte serait une réponse à l'abbé Claude François Houtteville, qui avait tenté d'établir La Vérité de la religion chrétienne prouvée par les faits (1722). Burigny reprend en effet à son compte le déplacement du débat opéré par l'abbé : la question ne réside plus dans l'examen spécifique et sans fin des dogmes mais dans l'étude critique des « faits de l'Évangile », qu'Houtteville présentait comme des preuves pour clore tout débat. En parallèle avec son frère Lévesque de Pouilly qui se consacra à une discussion critique concernant l'incertitude de l'histoire antique, Burigny s'attache à montrer combien les différents principes chargés de garantir la foi (l'Église, le libre examen ou le sentiment) sont incapables de satisfaire notre exigence rationnelle quant à l'exactitude desdits faits. Dès lors, la conclusion ne fait plus de doute : il faut sus- 
pendre son jugement faute de critère suffisant capable d'assurer une quelconque preuve factuelle.

$\mathrm{S}$. Landucci ne manque pas de faire remarquer, dans un appendice, combien l'influence de Pierre Bayle, mais du Bayle rationaliste et sceptique, permet d'éclairer ce texte. Dans le même appendice nous sont proposés d'autres extraits des querelles de la fin du XVII ${ }^{c}$ siècle, entre Pierre Nicole, Pierre Jurieu et Jean Claude d'une part, puis entre Pierre Jurieu et Élie Saurin d'autre part, qui avaient tenté de trouver un principe capable de guider dans la recherche de la religion même et surtout les simples, uitime marque de la vraie religion.

Le texte de Burigny, en même temps qu'il emprunte références et critiques à ces débats, les déplace et les transforme. En annonçant ainsi à travers les critiques qui lui seront faites (que recense un deuxième appendice) et les reprises dont il sera l'objet (Burigny fréquenta Voltaire) la reformulation des liens entre raison et foi, dans le sens d'une «dramatisation de ces rapports » comme le note S. Landucci. Burigny nous fait ainsi passer, après et grâce à Bayle, d'un siècle à l'autre.

La lecture de ce texte marque donc une étape importante du rationalisme, de l'histoire des emplois et des domaines dans lesquels la raison peut s'exprimer. Toutefois, il convient de remarquer un autre aspect intéressant de cet écrit de Burigny : la réflexion critique sur les faits historiques, fruit elle aussi d'un développement important à la fin du Grand Siècle mais dont la postérité sera bien moins grande en France. De ce point de vue, Burigny s'inscrit, à l'instar de son frère, dans le cadre des débats de l'Académie des inscriptions et belles-lettres, qui furent dominés par la personnalité de Nicolas Fréret, à qui fut d'ailleurs attribué faussement ce texte.

De l'Examen de la religion est par conséquent un texte dense, riche et carrefour, que l'édition, les notes et la bibliographie de S. Landucci contribueront utilement à mieux faire connaître tant elles constituent un excellent outil pour rentrer dans le vif des débats concernant foi et raison à cette époque à partir de cet auteur pivot mais méconnu. 